\title{
Mutational and Susceptibility Genetics Are Different Clinical Paradigms
}

During the past 2 years there have been several consensus conferences on diagnostic testing for Alzheimer's disease (AD), specifically related to the use of new genetic tests (Farrer et al., 1995; Relkin et al., 1996). Unfortunately there is an appearance of controversy between some of the published opinions and the empirical data (Saunders et al., 1996; Slooter et al., 1996). The clinical paradigm for $A D$ has changed from "ruling out" a small list of reversible and treatment causes of dementia to "ruling in" the diagnosis of $\mathrm{AD}$ using adjunctive genetic tests with a high positive predictive value for some patients (Roses \& Saunders, in press).

\section{MUTATIONAL DISEASES VERSUS SUSCEPTIBILITY POLYMORPHISMS}

Apolipoprotein E (APOE) genotyping, as an adjunct for the diagnosis of $A D$, measures universally distributed polymorphisms associated with varying relative risks. APOE risk estimates are based on populations, not on private familial risks. APOE is a true susceptibility locus with three common alleles that are distributed throughout the population in varying frequencies. Inherited combinations of those alleles (APOE genotypes) are associated with different relative risks and age of onset distributions for the onset of the common form of AD (reviewed in Roses, 1996). Thus, an infrequent or uncommon mutation in APOE does not cause AD, in contrast to rare disease-causing autosomal dominant mutation forms of AD.

Unfortunately, most physicians are still only marginally trained in genetics, especially if formal medical training was completed before this past decade of molecular genetic progress. However, even members of the genetics community need to adjust their perception to appreciate the differences between family-specific disease-causing mutations and population risks of susceptibility polymorphisms or risk factors.

Disease-causing gene mutations are limited to a relatively small number of people who inherit risk in a well-defined genetic pattern, e.g., autosomal dominant, $X$-linked recessive, etc. Susceptibility polymorphisms, like APOE, are inherited in some form by everyone. Thus every individual has a definable age-dependent risk, strongly influenced by his or her APOE genotype, with other genetic and environmental factors. APOE has three alleles that are distributed in the population: The proportion of each allele varies in different racial and ethnic series (Table 1). The basic biological association between APOE and AD is that each dose of $\varepsilon 4$ increases the risk and 
lowers the age-of-onset distribution, whereas $\varepsilon 2$ decreases risk and raises the age-of-onset distribution (Roses, 1996). The median age of onset for $\varepsilon 4 / \varepsilon 4$ individuals is below 70 years of age, whereas that of $\varepsilon 2 / \varepsilon 3$ individuals is over 90 years. On the basis of the inheritance of particular APOE genotypes, there can be more than 20 years' difference in median age of onset between $2 \%$ of the population carrying the $\varepsilon 4 / \varepsilon 4$ genotype and the $13 \%$ with $\varepsilon 2 / \varepsilon 3$.

\section{PREDICTION VERSUS DIAGNOSIS: TWO DISTINCT APPLICATIONS}

Because the age-of-onset distribution for each genotype extends over more than 40 years, it is currently impossible to predict which individual will develop AD early, and who will be spared at age 100 years. Thus there is little practical prediction that can be provided to any cognitively intact individual, except a currently nonquantitative increase or decrease in relative risk. On the other hand, for cognitively impaired patients with a differential diagnosis that includes possible or probable AD after evaluation for other treatable causes of dementia, the positive predictive value (PPV) of carrying an $\varepsilon 4$ allele for the diagnosis of $A D$ (confirmed by autopsy) is very high, greater than $97 \%$ in the multicenterCERAD series (Kakulas et al., 1996; Saunders et al.,
1996; Smith et al., 1996; Welsh-Bohmer et al., in press).

The true prevalence of pathologically verified $A D$ in any series is only known after death, at a time when the information is not clinically relevant or useful. In series of demented patients, the accuracy of $\mathrm{AD}$ diagnosis is not known for each individual, nor is the autopsyconfirmed prevalence of AD determined for the series that contains that individual. (Clinical diagnosis cannot be defined as a "gold standard" and assigned a PPV of $100 \%$ [Slooter et al., 1996].) A major advantage of using APOE genotyping is increased individual diagnostic accuracy early in the disease course. Accurate early diagnosis offers patients the opportunity to participate in their own care, allows stratification of patients for predictive and therapeutic drug trials, may provide a selection factor for using approved drugs, and provides an opportunity for family counseling. When used in a predictive mode, APOE genotyping may be abused for insurance or other population-based projections. Therefore, its clinical use is recommended only for diagnosis. APOE genotyping is relatively inexpensive and is useful as an adjunct for accurate diagnosis of $A D$ only when the $\varepsilon 4$ allele is present $(40 \%$ to $75 \%$ of $\mathrm{AD}$ cases, depending on the selection for age, race, or ethnicity). It must be stressed that the absence of an $\varepsilon 4$ allele does not rule out

TABLE 1. Distribution of APOE Genotypes (Roses, 1996)

\begin{tabular}{ccc}
\hline APOE Genotypes & \% of Population & \% of Sporadic AD \\
\hline$\varepsilon 4 / \varepsilon 4$ & $2 \%$ & $17 \%$ \\
$\varepsilon 3 / \varepsilon 4$ & $21 \%$ & $43 \%$ \\
$\varepsilon 3 / \varepsilon 3$ & $58 \%$ & $33 \%$ \\
$\varepsilon 2 / \varepsilon 3$ & $13 \%$ & $3 \%$ \\
$\varepsilon 2 / \varepsilon 4$ & $5 \%$ & $3 \%$ \\
$\varepsilon 2 / \varepsilon 2$ & $<1 \%$ & $<1 \%$ \\
\hline
\end{tabular}


AD at all: There are no "false negatives" in this paradigm. No $\varepsilon 4$ is no information for practical diagnostic uses.

A critical and frequently misunderstood difference between the use of susceptibility genes (a new paradigm) and mutational causes of disease (the common paradigm) is the nonapplication of any APOE genotype for prediction in normal individuals and for estimating risks of relatives. Suppose you are the sibling of a patient with Huntington disease (HD): Your risk before mutation testing is $50 \%$-yet your actual risk is either $100 \%$ or $0 \%$ depending upon whether or not you carry the HD mutation. With APOE, no genotype predicts certainty of disease and no genotype eliminates risk in siblings or in the general population.

APOE genotyping plays a useful, early adjunctive role in evaluating cognitively impaired patients for AD, but provides no "false negatives." Kukull and colleagues (1996) state that "considering either homozygous or heterozygous $\varepsilon 4$ genotype as a screen or diagnostic marker for AD would miss many true cases and could misclassify many normals as AD." Both are true because many $A D$ patients have no $\varepsilon 4$ allele, but a diagnostic use in "normals" has never been suggested. Its use has been limited to the differential diagnosis of cognitively impaired patients. In many statements concerning the use of susceptibility polymorphisms, the prediction mode for "normals" is often blurred and confused with diagnostic applications for symptomatic patients. This develops from experience with mutational diseases, but is a different paradigm for susceptibility variants. Differential diagnosis is performed for individuals with appropriate symptoms and signs, in this case progressive cogni- tive impairment usually with memory problems, not for the general population. The a priori risks for $A D$ in demented patients versus the unaffected population are quite different, especially because more than half of patients with dementia will ultimately have autopsyconfirmed AD. Diagnostic and predictive uses are therefore two separate and distinct questions for susceptibility genes, an epidemiological paradigm rather than the more customary genetics of rare mutations (Roses, in press). AD is a complex disease paradigm in which everyone carries risk, with $\mathrm{APOE}$ a strong factor but not the only determinant. Education regarding the differences between mutational and susceptibility paradigms should be a mandatory first step before consensus or individual opinions for medical practice are enunciated.

Allen D. Roses, MD Center for Human Genetics Departments of Medicine (Neurology) and Neurobiology Duke University Medical Center Durham, NC, USA

\section{REFERENCES}

Farrer, L. A., Brin, M. F., Elsas, L., Goate, A., Kennedy, J., et al. (1995). Statement on use of apolipoprotein $\mathrm{E}$ testing for Alzheimer disease. American College of Medical Genetics / American Society of Human Genetics Working Group on ApoE and Alzheimer disease. Journal of the American Medical Association, 274, 1627-1629.

Kakulas, B. A., Wilton, S. D., Fabian, V. A., \& Jones, T. M. (1996). Apolipoprotein-E genotyping in the diagnosis of Alzheimer's disease in an autopsy confirmed series. Lancet, 348, 483.

Kukull, W. A., Schellenberg, G. D., Bowen, J. D., McCormick, W. C., Yu, C. E., et al. 
(1996). Apolipoprotein E in Alzheimer's disease risk and case detection: $A$ case-control study. Journal of Clinical Epidemiology, 49, 1143-1148.

Relkin, N. R., Tanzi, R., Breitner, J., Farrer, L., Gandy, S., et al. (1996). Apolipoprotein E genotyping in Alzheimer's disease. Lancet, 347, 1091-1095.

Roses, A. D. (1996). Apolipoprotein E alleles as risk factors in Alzheimer disease. Annual Review of Medicine, 47, 387-400.

Roses, A. D. (in press). A new paradigm for clinical dementia evaluations: Alzheimer disease and apolipoprotein $\mathrm{E}$ genotypes. In S. G. Post \& P. J. Whitehouse (Eds.), Ethics, genetics and Alzheimer disease. Baltimore: Johns Hopkins University Press.

Roses, A. D., \& Saunders, A. M. (in press). Apolipoprotein $\mathrm{E}$ genotyping as a diagnostic adjunct for Alzheimer's disease. International Psychogeriatrics, 9(Suppl. 1).
Saunders, A. M., Hulette, C., Welsh, K. A., Schmechel, D. E., Crain, B., et al. (1996). Specificity, sensitivity and predictive value of apolipoprotein E genotyping for sporadic Alzheimer's disease. Lancet, 348, 90-93.

Slooter, A. J., Breteler, M. M. B., Ott, A., Van Broeckhoven, C., \& van Duijn, C. $M$. (1996). APOE genotyping in differential diagnosis of Alzheimer's disease. Lancet, 348, 334.

Smith, A. D., Jobst, K. A., Johnston, C., Joachim, C., \& Nagy, Z. (1996). Apolipoprotein-E genotyping in diagnosis of Alzheimer's disease. Lancet, 348, 483-484.

Welsh-Bohmer, K. A., Gearing, M., Saunders, A. M., Roses, A. D., \& Mirra, S. (in press). Apolipoprotein E genotypes in a neuropathological series from the Consortium to Establish a Registry for Alzheimer's disease (CERAD). Annals of Neurology.

\section{\$ Springer Publishing Company Treating Alzheimer's and Other Dementias}

\section{Manfred Bergener, MD, and Sanford I. Finkel, MD, Editors}

This volume's international scope provides a comprehensive overview of the most current knowledge on Alzheimer's Disease. Based on the 6th Congress of the International Psychogeriatrics Association, the prestigious editors have included contributions ranging from prevention, diagnosis, and treatment to the latest activities in clinical Alzheimer's research.

Partial Contents: Further Developments in the Molecular Biology of Alzheimer's Disease with Special Reference to the Development of New Methods of Treatment - Latest Advances in Alzheimer's Drug Research • Electroconvulsive Therapy for Agitated Dementia Patients $\bullet$ Social Ties and Social Support Among Alzheimer's Disease Caregivers

$$
1995 \quad 576 p p \quad 0-8261-8930-X \text { hardcover } \$ 61.95 \text { (outside US } \$ 68.50 \text { ) }
$$

536 Broadway, New York, NY 10012-3955 • (212) 431-4370 • Fax (212) 941-7842 